\title{
Failure to vaccinate against whooping cough
}

\author{
D STEVENS, R BAKER, AND S HANDS \\ Gloucestershire Royal Hospital, Gloucester, and The Leckhampton Surgery, Cheltenham, Gloucestershire
}

SUMMARY We describe a prospective study in which we investigated why children fail to get vaccinated against whooping cough, including an assessment of the attitudes of parents and professionals and the impact of different views of the contraindications. There was considerable disagreement among the professionals on the interpretation of the contraindications to immunisation, and the commonest reason for omitting pertussis vaccine was advice from the doctor based on dubious contraindications. When faced with parents anxious about the risks of immunisation health professionals are unable to find reassurance in the list of contraindications to immunisation.

After the publicity given to a report linking pertussis vaccination with neurological damage ${ }^{1}$ the uptake of the vaccine fell from around $80 \%$ in 1974 to $31 \%$ in $1978 .^{2}$ Doctors have been encouraged to continue to vaccinate children against whooping cough but warned about the importance of observing the contraindications. ${ }^{3}$ Unfortunately, there is a lot of confusion about the interpretation of the contraindications. ${ }^{4}$ We describe a prospective study in which we investigated why children fail to get vaccinated against whooping cough, including an assessment of the views of parents and health professionals and the impact of different views of the contraindications.

\section{Method}

The Gloucestershire child health computer records for 1982 showed that for children born in 1979, although the average uptake for three courses of diphtheria, tetanus, pertussis (DTP) immunisations was $52 \%$, in some general practices more than $60 \%$ were immunised against whooping cough ('high uptake'), whereas in others ('low uptake') the figure was less than $40 \%$. Roughly half the general practices in Gloucestershire fell into these two categories. We followed up all babies born during a three month period (April, May, and June 1983) who were registered with these practices. After the first visit for immunisation the parent who brought the child (the mother in all cases) was asked to complete a structured questionnaire by the health visitor. The mother was asked about the reasons for the decision about whooping cough vaccination, who helped her make the decision, and what advice was given. At the same time, the mother's views on the risks of serious side effects of whooping cough vaccination, the effectiveness of the vaccine, the severity of whooping cough infection, and the risks of catching whooping cough if not vaccinated were assessed by graded questions (Fig. 1).

Immediately after completion of the parents survey, questionnaires were sent to general practitioners, health visitors, and clinical medical officers involved in immunising children from these practices.

The views of these health professionals on whooping cough vaccination and whooping cough infection were assessed with the same graded questions answered by the mothers (Fig. 1), and in addition their interpretation of contraindications was assessed by 44 short case histories in which the child was stated to be well except for the problem outlined and the parents willing to take the advice of the doctor or health visitor. Questions on related topics-for example, history of allergy in the child or members of the family-were distributed at random throughout the questionnaire. The $\chi^{2}$ test and Student's $t$ test were used for statistical comparison.

Fig. 1 (opposite) Views of health professionals and mothers on (a) risks of serious side effects of whooping cough vaccination, (b) effectiveness of vaccine at preventing whooping cough, (c) seriousness of whooping cough infection, and (d) chances of catching whooping cough if not vaccinated.

$\mathrm{GPs}=$ general practitioners; $\mathrm{CMOs}=$ clinical medical officers; $\mathrm{DTP}=$ diphtheria, pertussis, and tetanus; $\mathrm{DT}=$ diphtheria and tetanus. 
Failure to vaccinate against whooping cough 383
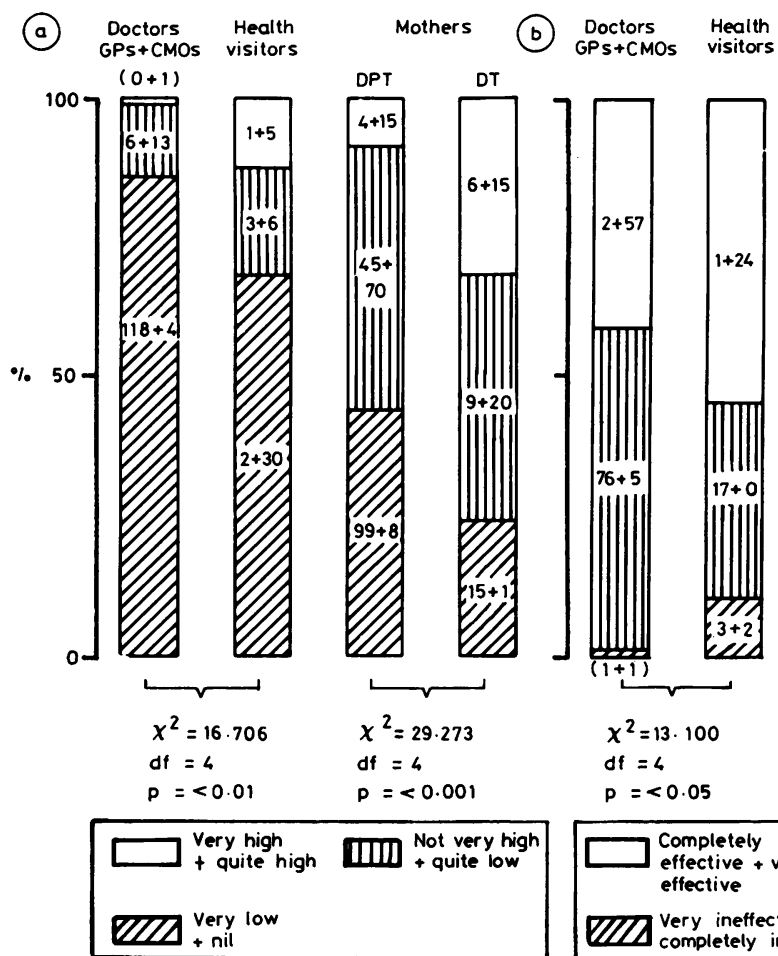

Mothers

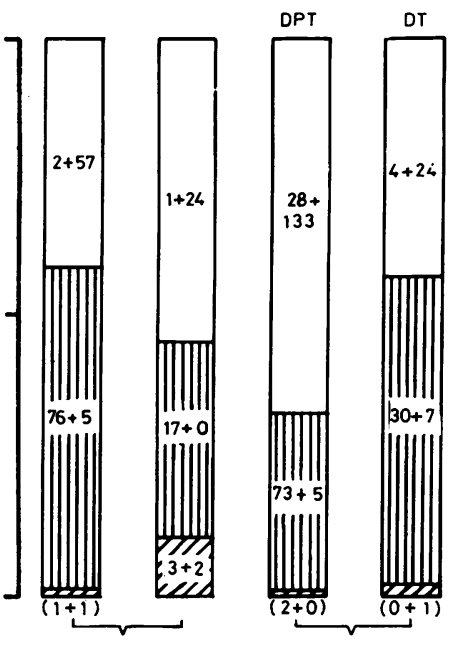

$x^{2}=13.100$

$x^{2}=22.523$

$\mathrm{df}=4$

of $=4$

$p=<0.05$

$p=<0.001$

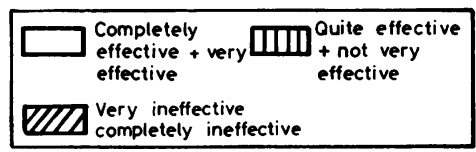

(c)

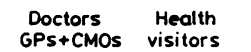

Mothers

(d) $\begin{gathered}\text { Doctors } \\ \text { GPs+CMOs }\end{gathered}$ Health
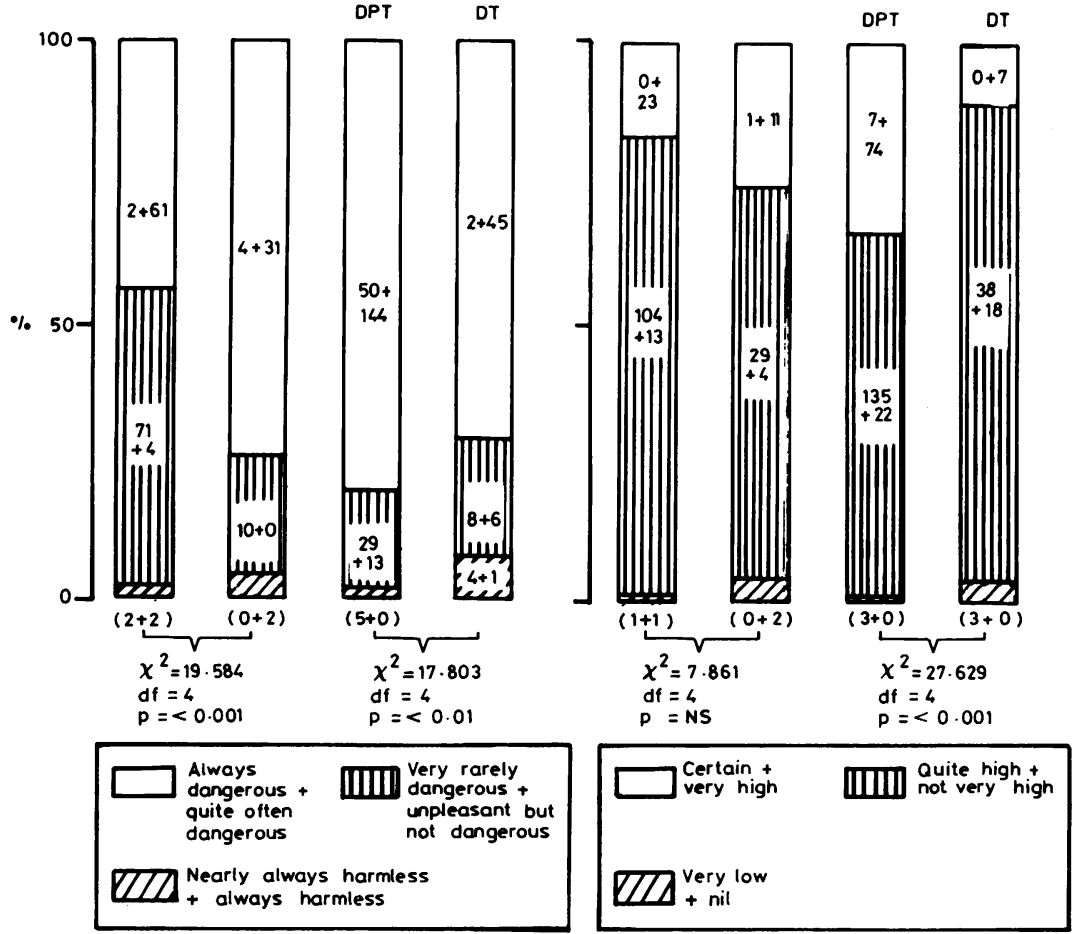


\section{Results}

Uptake of DTP immunisation. We were able to follow up $315(87 \%)$ of the 362 babies. In the "high uptake' practices $105(81.4 \%)$ had DTP and 24 DT only, whereas in the 'low uptake' practices 136 (73.1\%) had DTP and 42 DT only, and eight had no immunisations at all.

Details of mothers and infants.

Comparison of infants who were given DTP $\mathrm{v}$ those given $D T$ only

There was no significant difference in social class, number of siblings, place of immunisation (surgery or child health clinic) and mother's marital state. Mothers whose infants were given DTP tended to be older (mean (SD) age 27.5 (4.89) years $v 25 \cdot 8(4 \cdot 64)$ years, $p=<0 \cdot 01)$ and regarded the vaccine as more effective $(p=<0.001)$, the risks of serious side effects of vaccination as less $(p=<0.001)$ and whooping cough infection as more serious $(p=<0 \cdot 01)$, and thought there was a greater chance of catching the disease if the vaccine was not given $(\mathrm{p}=<0 \cdot 001)$ (Fig. 1).

Comparison of infants registered with 'high uptake' and 'low uptake' practices

There was no significant difference with regard to any of the above factors except that a significantly higher proportion of infants from 'high uptake' practices attended a surgery rather than a child health clinic for immunisation ('high uptake' practices-surgery:clinic ratio=95:34; 'low uptake' practices-79:106 $(\mathrm{p}=<0 \cdot 001)$.

Health professionals survey. All 58 of the general practitioners and $96 \%$ (26 out of 27 ) of the health visitors from 'high uptake' practices, $96 \%$ (46 out of 48 ) of the general practitioners and $91 \%$ (21 out of 23 ) of the health visitors from 'low uptake' practices, and $97 \%$ (36 out of 37) of the clinical medical officers completed the questionnaire. Many of the clinical medical officers were working in clinics attended by children from both 'high uptake' and 'low uptake' practices. The results of their questionnaires were therefore considered together.

Views of general practitioners, clinical medical officers, and health visitors on whooping cough vaccination and whooping cough infection

Comparison between 'high uptake' general practitioners, 'low uptake' general practitioners, and clinical medical officers showed no significant differences in their views on whooping cough vaccination and whooping cough infection. Neither did 'high uptake' and 'low uptake' health visitors show any significant differences in their views on these matters. When all the doctors were grouped together and compared with all the health visitors (Fig. 1) the doctors were less concerned about the risks of serious side effects of vaccination $(p=<0 \cdot 01)$ but were also less concerned about the seriousness of whooping cough infection $(\mathrm{p}=<0.01)$, and the health visitors were less convinced about the effectiveness of the vaccine $(p=<0 \cdot 05)$. There was no significant difference between doctors and health visitors in their assessment of the chances of a non-immunised child catching whooping cough.

Views of general practitioners, clinical medical officers, and health visitors on the contraindications to whooping cough vaccination

In only two out of the 44 questions did more than $90 \%$ of the health professionals within each group agree on the correct answer. The proportion of 'high uptake' general practitioners opting to immunise was greater than both 'low uptake' general practitioners and clinical medical officers in 37 out of 44 $(84 \%)$ of the questions. The proportion of high uptake' health visitors opting to immunise was greater than 'low uptake' health visitors in 40 out of $44(91 \%)$ of the questions. The results of some individual questions are given in Table 1.

Reasons for omitting whooping cough vaccine

Table 2 summarises the reasons for omitting whooping cough vaccine and indicates the source of advice to parents. The commonest reason for omitting the whooping cough vaccine was a family history of neurological disorder or fits. Doctors were the main source of advice when the child was excluded because of contraindications. The source of the mother's concern about the vaccine was attributed more often to the influence of a relative or acquaintance than to the press or television.

Table 1 shows the individual contraindications that excluded children from whooping cough vaccination and the response that health professionals gave when asked about the equivalent problem in the questionnaire.

\section{Discussion}

The rise in pertussis immunisation uptake between 1979 and 1983 is reflected in these figures. Moreover, the gap between high and low uptake practices has narrowed, although it has persisted. For example, for Gloucestershire children born in 1981, $25 \%$ of practices had an uptake below $60 \%$, while a 


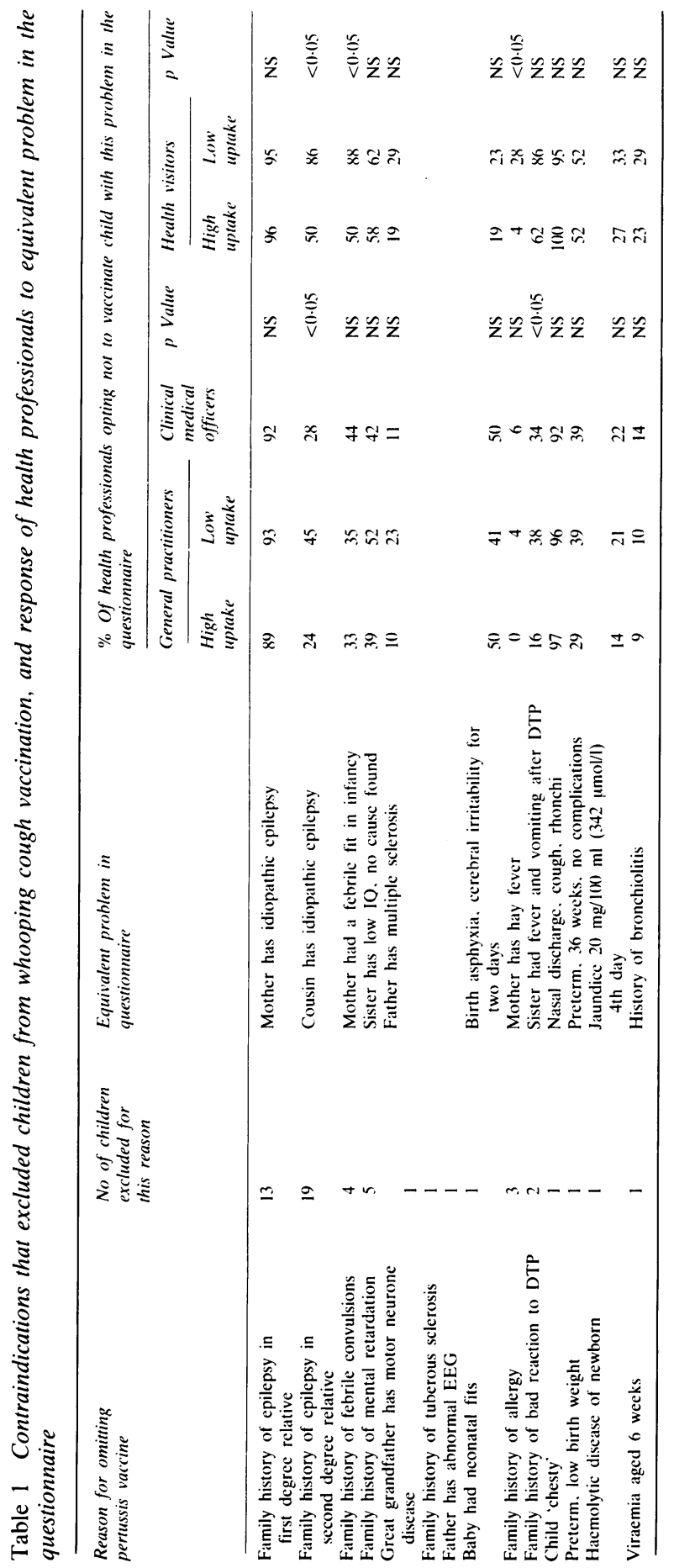


Table 2 Reasons given for omitting pertussis vaccine and who the advice to the parents was given by

\begin{tabular}{|c|c|c|c|c|c|c|}
\hline \multirow[t]{2}{*}{ Reason } & \multicolumn{6}{|c|}{ Source of advice } \\
\hline & Doctor ${ }^{*}$ & $\begin{array}{l}\text { Health } \\
\text { visitor }\end{array}$ & $\begin{array}{l}\text { Relative/ } \\
\text { acquaintance }\end{array}$ & Media & $\begin{array}{l}\text { Mother's } \\
\text { own decision }\end{array}$ & Total \\
\hline Family history of central nervous system problems and fits & 27 & 5 & 4 & - & 5 & 41 \\
\hline Miscellaneous contraindications & 5 & 1 & 3 & - & 5 & 14 \\
\hline $\begin{array}{l}\text { Concern about side effects of vaccine, ineffectiveness of } \\
\text { vaccine, or pertussis not serious }\end{array}$ & - & - & 12 & 4 & 9 & 25 \\
\hline
\end{tabular}

*Includes general practitioners and clinical medical officers

In 14 children there was more than one reason.

further $25 \%$ had an uptake of $76 \%$ or above. Some caution may be needed when drawing conclusions from the results of the parents' questionnaire, as this was administered by health visitors, although the questionnaire was structured so as to avoid problems arising from this source. This section of the study largely provided negative information, ruling out social factors as determinants of immunisation acceptance.

Mothers whose infants were not given whooping cough vaccine were less convinced about the need for vaccination and more worried about the risks of serious side effects of vaccination than mothers whose infants were given DTP. The commonest reason given, however, for giving DT rather than DTP was advice from the doctor that the pertussis component should be omitted because of a family history of neurological disorder or fits. This and other dubious contraindications were commoner reasons for omitting whooping cough vaccine than mothers' fears about the vaccine or lack of conviction about its need.

The differences in the interpretation of contraindications between the health professionals vaccinating children from the two types of practice probably contributes to the different proportion of children vaccinated against whooping cough in these practices. The reasons for the differences in interpretation of contraindications are not clear but are not due to different views on the risks or importance of vaccination. The instructions lack clarity about relative contraindications and ask doctors to exercise their judgment in each case. It is not surprising that this leads to a wide variety of decisions.

Part of the Department of Health and Social Security (DHSS) instructions reads as follows: 'There are certain groups of children in whom whooping cough vaccination is not absolutely contraindicated but who require special consideration as to its advisability. These groups are (1) children whose parents or siblings have a history of idiopathic epilepsy; (2) children with developmental delay thought to be due to a neurological defect; and (3) children with neurological disease. For these groups the risks of vaccination may be higher than in normal children but the effects of whooping cough may be more severe, so that the benefits of vaccination would also be greater. The balance of risk and benefit should be assessed with special care in each individual case. Allergy, according to a substantial body of medical opinion, is not a contraindication to the administration of pertussis vaccine, but doctors should use their own discretion in the individual case. ${ }^{3}$ The advice about relative contraindications and allergy is expressed in terms that suggest that it may be advisable to vaccinate these children without giving reassurance that it is safe to do so. Further difficulties are introduced if the manufacturers' instructions are referred to, where it will be found that a family history of epilepsy (not specifying first degree relatives) and severe allergy are listed as absolute contraindications. $^{5}$

The DHSS instructions are introduced by a warning in italics 'it is extremely important that the contraindications to whooping cough vaccination should be observed'. With the background of this warning and the likelihood of recrimination if a neurological problem came to light or developed after vaccination, it is not surprising that many doctors exclude children from vaccination on the basis of any possible contraindication mentioned in the DHSS or manufacturers' instructions. The wording of the contraindications makes it easy for doctors to exclude children from vaccination on the basis of dubious contraindications when faced with parents who are anxious about the vaccine.

Our study was only concerned with the first DTP immunisation. Figure 2 shows the response of health professionals when a child develops a temporary reaction after the first DTP immunisation. Reactions similar to this were found to occur in more than a third of children after the first DTP immunisation, ${ }^{6}$ and clearly many health professionals would exclude a child from completing a course of DTP immunisations after such a reaction. 


\section{General practitioners}

\section{'High uptake' \\ 'Low uptake'}

Clinical medical officers

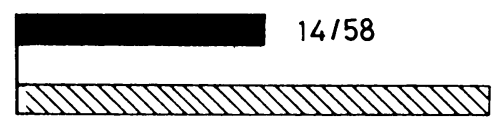

$10 / 26$

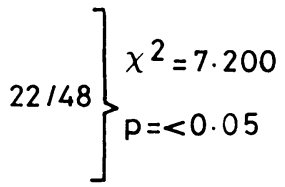

\section{Health visitors}

'High uptake'

'Low uptake'
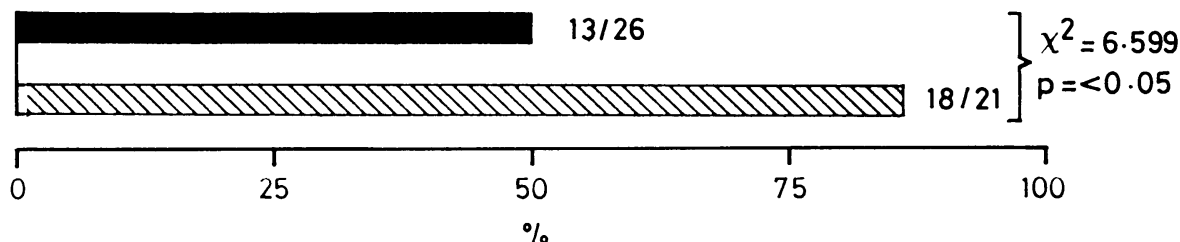

Fig. 2 Percentage of health professionals who would not give a further dose of DTP if there was a temporary reaction (soreness of buttock (I inch) or fever $38^{\circ} \mathrm{C}$ for 24 hours) after the first dose.

The introduction of newer vaccines ${ }^{7}$ with fewer side effects of this nature could be expected to increase the number of children completing a full course of DTP immunisations. Until such time the instructions should be more specific about which reactions should exclude children from further courses of DTP.

The results of our survey suggest that improvement in the uptake of whooping cough vaccine could be achieved (1) if parents and the public at large were persuaded that the vaccine is safe, effective, and necessary; (2) if the instructions of the DHSS and of the manufacturer were simplified, expressed identically and clearly, and contained positive reassurance that it is safe and desirable to vaccinate children with some problems that are at present used as contraindications; and (3) if a vaccine with fewer immediate reactions was introduced.

We are grateful for the help given by Professor J Colley and Dr R Midwinter of the Department of Community Medicine. University of Bristol. Miss M Bevan of the Gloucestershire Area Health
Authority, Mrs J Bunt of Gloucestershire Royal Hospital, and to all those who completed the questionnaires.

\section{References}

1 Kulenkampff M, Schartzman JB, Wilson J. Neurological complications of pertussis inoculation. Arch Dis Child 1974:49:46-9.

2 Anonymous. Pertussis vaccine [Editorial]. Br Med J 1981: 282:1563-4.

- Department of Health and Social Security. Immunisation against infectious disease-whooping cough. London: DHSS, 1984.

${ }^{4}$ Hull D. Interpretation of the contraindications to whooping cough vaccination. Br Med J 1981:283:1231-3.

Wellcome Research Laboratories. Trivax Diphtheria Tetanus and Pertussis Vaccine BP (I)T Per/Vac). Beckenham, England: Wellcome Research Laboratories. 1984.

' Cody CL, Barraff LJ. Cherry JD, et al. Nature and rates of adverse reactions associated with DTP and DT immunisations in infants and children. Pediatrics 1981:68:650-60.

Sato Y. Kimura M. Fukumi H. Development of a pertussis component vaccine in Japan. Lancet 1984;i:122-6.

Correspondence to Dr R H Baker. The Leckhampton Surgery, 'Lloyd Davies House'. 17 Moorend Park Road, Cheltenham GL3 OLA. Gloucestershire, England.

Received 8 January 1986 